\title{
Pfannenstiel vs. midline incision for urinary diversion, following minimally invasive radical cystectomy: single center experience
}

\author{
Gopal Ramdas Tak, Arvind P. Ganpule, Abhishek G. Singh, Aditya Pratap Singh Sengar, Mohankumar \\ Vijayakumar, Sudharsan S. Balaji, Ravindra B. Sabnis, Mahesh R. Desai
}

Department of Urology, Muljibhai Patel Urological Hospital (MPUH), Nadiad 387001, Gujarat, India.

Correspondence to: Dr. Gopal Ramdas Tak, Department of Urology, Muljibhai Patel Urological Hospital, Dr. V V Desai Road, Nadiad 387001, Gujarat, India. E-mail: drgopaltak@gmail.com

How to cite this article: Tak GR, Ganpule AP, Singh AG, Sengar APS, Mohankumar V, Balaji SS, Sabnis RB, Desai MR. Pfannenstiel $v s$. midline incision for urinary diversion, following minimally invasive radical cystectomy: single center experience. Mini-invasive Surg 2018;2:15. http://dx.doi.org/10.20517/2574-1225.2018.05

Received: 26 Jan 2018 First Decision: 23 Mar 2018 Revised: 23 Apr 2018 Accepted: 8 May 2018 Published: 31 May 2018

Science Editor: Charles F. Bellows Copy Editor: Jun-Yao Li Production Editor: Cai-Hong Wang

\begin{abstract}
Aim: The present study is to assess the morbidity on comparing Pfannenstiel vs. midline incision following minimally invasive radical cystectomy.
\end{abstract}

Methods: This is a retrospective comparative study from February 2004 to February 2017 and the number of patients
studied was 116. Patients were divided into group A (Pfannenstiel incision) and group B (midline incision). The parameters
analyzed were age, gender, co-morbidity, tobacco exposure, occupation, presentation, computed tomography findings,
hydronephrosis, transurethral resection of bladder tumor report, duration of surgery (in minutes), hemoglobin drop (in
gram per deciliter), need for blood transfusion (number of units), hospital stay (in days), epidural analgesia, analgesic
requirement, pain score on first three postoperative days (on visual analogue scale), complications, and lymph node
yield (numbers). Standard steps included cystectomy with bilateral pelvic lymph-adenectomy done either through the
laparoscopic or robotic approach and specimen retrieval along with diversion through either Pfannenstiel or midline incision.

Results: Primary end points, post operative pain score $(P=0.0001)$, analgesic requirement $(P=0.0003)$, post operative wound complication $(P=0.002)$, length of hospital stay $(P=0.0003)$ all were less (statistically significant $P<0.05)$ for group $A$ as compared to group $B$ and secondary end points, duration of surgery $(P=0.0002)$, post operative paralytic ileus duration $(P=0.0006$ ) were less (statistically significant $P<0.05$ ) for group A as compared to group B. Other secondary end points, post operative hemoglobin drop $(P=0.08)$, the number of units of blood transfused $(P=0.189)$ and lymph node yield $(P=0.533$ ) were comparable in either group (statistically insignificant $P \geq 0.05$ ).

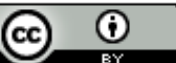

(C) The Author(s) 2018. Open Access This article is licensed under a Creative Commons Attribution 4.0 International License (https://creativecommons.org/licenses/by/4.0/), which permits unrestricted use, sharing, adaptation, distribution and reproduction in any medium or format, for any purpose, even commercially, as long as you give appropriate credit to the original author(s) and the source, provide a link to the Creative Commons license, and indicate if changes were made. 
Conclusion: Minimally invasive (laparoscopic or robotic) radical cystectomy with an extra-corporeal diversion through Pfannenstiel incision offers an advantage of less morbidity than midline incision.

Keywords: Pfannenstiel, midline, incision, minimally invasive radical cystectomy, conduit, neobladder, morbidity

\section{INTRODUCTION}

Minimal access radical cystectomy is now commonly performed for localized carcinoma bladder but the reconstructive part of surgery is challenging. Open reconstruction can offer the advantage of minimal access surgery (MAS). Morbidity of open surgery can be reduced and the advantage of MAS can be gained with Pfannenstiel incision, transverse incisions in abdominal surgery are based on better anatomical and physiological principles ${ }^{[1]}$. In comparison with other treatment options, radical cystectomy offers better local disease control and 5-year survival.

Usually, radical cystectomy has become the part of multimodality of treatment of bladder cancer in select cases (in which neo-adjuvant chemotherapy is followed by radical cystectomy). Approaches for radical cystectomy are open, laparoscopic or robotic; and "open" approach is the gold standard of surgical treatment. Even in this era of minimally invasive surgery, there are some institutions/centers, which practice the "open" approach. Open approach has its own disadvantages like intra-operative blood loss, prolonged hospital stay, delay in recovery, significant morbidity and even mortality. But now, in the era of minimal access surgery, laparoscopy and robotic approaches can become standard of care for the surgical management of organ-confined carcinoma bladder with advantages of less blood loss, high lymph node yield, less pain, early recovery, fewer complications and mortality and short hospital stay. The major disadvantages of minimal access surgical approach are costs of implementation and lack of haptic feedback.

This study aims to assess the morbidity on comparing Pfannenstiel $v s$. midline incision for urinary diversion, following minimally invasive radical cystectomy.

\section{METHODS}

This is a retrospective comparative study from February 2004 to February 2017 and the number of patients studied was 116. Patients were divided into group A (Pfannenstiel incision) and group B (midline incision). All operations were performed by the single surgeon with experience of doing about 20 radical cystectomies per year. The incision was not allocated by any random chart or table as it is a retrospective study. The allocation was based on surgeon's preference.

We used minimal access approach for radical cystectomy. Standard steps included transperitoneal approach. 5 ports for laparoscopy and 6 ports for robotic surgery were employed. Steps were bilateral ureter dissection, posterior peritoneotomy, bilateral seminal vesicles and vas dissection, dissection of the prostate, while cystoprostatectomy specimen was freed all around, specimen bagged, followed by bilateral standard lymph node dissection and initiation of the reconstructive part. We have not used staplers for any reconstruction due to financial constraints. The procedure was completed in two stages for ileal conduit: first, pelvic lymphadenectomy with cystoprostatectomy; second, extracorporeal formation of the ileal conduit. The procedure was completed in three stages for ileal neobladder: initially, pelvic lymphadenectomy with cystoprostatectomy, secondly, extracorporeal formation of neobladder and thirdly, re-docking the robot, intra-corporeal urethro-neo-vesical anastomosis.

For reconstructive part of the procedure (either an ileal conduit or ileal neobladder), we used lower midline vertical incision (group B) or Pfannenstiel incision (group A); we divided the patients into two groups. One was with lower midline vertical incision and the other with Pfannenstiel incision. We compared 
Table 1. Gender distribution of study population

\begin{tabular}{lccc}
\hline & Male & Female & Total \\
\hline Group A & 46 & 7 & 53 \\
Group B & 57 & 6 & 63 \\
\hline
\end{tabular}

various variables between these groups to assess the impact on outcomes. No patient was given neoadjuvant chemotherapy.

Parameters analyzed were age (in years), gender, co-morbidity, history of smoking or tobacco chewing, occupation, presentation, computed tomography findings, hydronephrosis, trans urethra resection of bladder tumor report, duration of surgery (in minutes), hemoglobin drop (in gram per deciliter), need for blood transfusion (number of units), hospital stay (in days), epidural analgesia, analgesic requirement (I.V. Tramadol was the only analgesic used, in milligrams of tramadol, no narcotic used in any patient), pain score on first three post operative days (on visual analogue scale), complications, wound complications specifically mentioned needing conservative management or secondary re-suturing (dehiscence and burst abdomen), delayed follow up, and lymph node yield (numbers). Standard steps were cystectomy with bilateral pelvic lymphadenectomy done either through the laparoscopic or robotic approach and specimen retrieval along with diversion through either Pfannenstiel or midline incision.

A midline incision begins with the vertical midline skin incision extending from pubic symphysis to just above the umbilicus; next, the sheath was opened vertically and then peritoneum was reached. A pfannenstiel incision begins with the transverse skin incision along the skin crease or 2-3 fingers above pubic symphysis extending to lateral borders of rectus muscle on either site, and then the rectus sheath was opened vertically to reach the peritoneum.

Primary endpoints in this study were postoperative pain score, analgesic requirement, postoperative wound complications, length of hospital stay and secondary endpoints were duration of surgery, postoperative paralytic ileus duration, postoperative hemoglobin drop, the number of units blood transfused, and lymph node yield.

We used a student's $t$-test for equality of means to compare various variables between the two groups. The analysis done was multivariate analysis.

\section{RESULTS}

The gender distribution of two groups has been shown in Table 1.

We have compared group A and group B with especial focus on various variables [Table 2]. We found that there was statistically significant $(P<0.0006)$ difference with regards to mean operative time which was 259 min for group A and 416 min for group B. This significant difference might be due to use of the midline vertical incision during initial learning curve period. The mean postoperative hemoglobin drop for group A was $1.75 \mathrm{~g}$ per deciliter; for group B it was $1.9 \mathrm{~g}$ per deciliter, which was statistically insignificant $(P=0.08)$. There was higher mean lymph node yield in group A 22.27 compared to group B which had 20.74, but it was not statistically significant $(P=0.533)$. The mean number of blood transfusions unit needed for group A (0.74) was lower than that for group B (1.00), but it was not statistically significant $(P=0.189)$.

We found that there was a statistically significant $(P=0.000)$ difference with regards to mean postoperative pain score on day 2 and day 3 between the studied groups, but the mean postoperative pain score for day 1 was insignificant for these groups. Overall there was a significantly lower pain score in group A than group B. Accordingly analgesic requirement (patients were administered injection tramadol intravenously, no additional regional/ epidural/systemic analgesia was given to any patient) was statistically significantly lower in group A as 
Table 2. Comparison among the study groups for various variables

\begin{tabular}{llll}
\hline Variable & Group A & Group B & P value \\
\hline Duration of surgery (min) & $259 \pm 72.14$ & $416 \pm 183.03$ & 0.0006 \\
Hb drop (gram per deciliter) & $1.75 \pm 0.71$ & $1.9 \pm 0.87$ & 0.08 \\
Blood transfused (units) & $0.74 \pm 1.01$ & $1.00 \pm 1.10$ & 0.189 \\
Post op pain score day-1 & $6.03 \pm 0.27$ & $7.9 \pm 0.35$ & 0.37 \\
Post op pain score day-2 & $5.18 \pm 0.99$ & $6.0 \pm 0.0$ & 0 \\
Post op pain score day-3 & $4.03 \pm 0.27$ & $5.0 \pm 1.0$ & 0 \\
Post op pain score overall & $5.08 \pm 0.66$ & $6.3 \pm 0.86$ & 0.0001 \\
Analgesic need (milligram of tramadol) & $642.45 \pm 145.27$ & $915.07 \pm 137.85$ & 0.0003 \\
Post op ileus (days) & $3.40 \pm 0.96$ & $4.61 \pm 1.09$ & 0.0006 \\
Hospital stay (days) & $10.16 \pm 3.39$ & $13.63 \pm 5.37$ & 0.004 \\
Lymph node yield & $22.27 \pm 14.32$ & $20.74 \pm 13.57$ & 0.533 \\
Age & 61.32 & 60.84 & 0.3 \\
\hline
\end{tabular}

Table 3. Various complications among the two groups

\begin{tabular}{lcc}
\hline Complication & Group A & Group B \\
\hline Ileus for 3 days & 42 & 12 \\
Ileus for 4 days & 7 & 17 \\
Ileus for 5 days & 3 & 14 \\
Ileus for 6 days & 1 & 1 \\
Ileus for 7 days & 0 & 1 \\
Wound infection conservative management & 5 & 13 \\
Wound infection secondary re-suturing & 3 & 9 \\
Pelvic collection & 1 & 0 \\
Acute Respiratory Distress Syndrome (ARDS) & 2 & 2 \\
Pneumo-thorax & 0 & 1 \\
Myocardial ischemia & 0 & 1 \\
Acute gastric dilatation & 1 & 0 \\
Bowel anastomotic leak & 1 & 0 \\
Ureter anastomotic leak & 0 & 3 \\
Rectal injury (de-functioning colostomy & 0 & 1 \\
Death & 2 & 3 \\
\hline
\end{tabular}

compared to group B. It was $642.45 \mathrm{mg}$ of tramadol and $915.07 \mathrm{mg}$ of tramadol for group A and group B respectively, showing a significant difference $(P=0.0003)$.

The mean hospital stay for group A was 10.16 days and 13.16 for group B, varying significantly $(P=0.004)$. We have compared these two groups in relation to complications too [Tables 3 and 4]. Postoperative ileus was defined as hypo motility of the gastrointestinal tract in the absence of mechanical bowel obstruction. We have found the mean duration of postoperative paralytic ileus for group A to be 3.4 days and 4.61 days for group $\mathrm{B}$; the difference was statistically significant $(P=0.001)$ [Table 2].

There were more wound-related complications in group B (22 out of 63 patients; 41\%) than group A ( 6 out of 53 patients; $15 \%)$, showing a statistically significant difference $(P=0.008)$. As we observed, there were more overall complications in group B (30 out of 63 patients; $46 \%$ ) as compared to group A (13 out of 53 patients; $24 \%$ ). Complications rate was significantly higher for group $\mathrm{B}(P=0.006)$.

One patient with a body mass index of $33 \mathrm{~kg} / \mathrm{m}^{2}$ in Pfannenstiel group had bowel leak and 3 patients of midline incision group had urine leak from the site of anastomosis, but it was statistically insignificant $(P=0.17)$.

We have compared mortality data between these groups and found that 2 out of 53 (3.7\%) patients in group A died, while 3 out of $63(4.7 \%)$ patients in group B died. Though there was more mortality in group B, it was not statistically significant $(P=0.47)$. 
Table 4. Level of significance of complications among the two groups

\begin{tabular}{|c|c|c|c|c|c|c|c|c|c|c|}
\hline Complications & $\begin{array}{l}\text { Ileus } \leq \\
3 \text { days }\end{array}$ & $\begin{array}{l}\text { Ileus }> \\
3 \text { days }\end{array}$ & $\begin{array}{l}\text { Wound } \\
\text { infection }\end{array}$ & $\begin{array}{l}\text { No wound } \\
\text { infection }\end{array}$ & $\begin{array}{c}\text { Anastomotic } \\
\text { leak }\end{array}$ & $\begin{array}{c}\text { No anastomotic } \\
\text { leak }\end{array}$ & $\begin{array}{c}\text { All } \\
\text { complications }\end{array}$ & $\begin{array}{c}\text { No } \\
\text { complications }\end{array}$ & Death & $\begin{array}{c}\text { No } \\
\text { death }\end{array}$ \\
\hline Group A & 42 & 11 & 8 & 45 & 1 & 3 & 13 & 40 & 2 & 51 \\
\hline Group B & 12 & 51 & 22 & 41 & 3 & 60 & 30 & 33 & 3 & 60 \\
\hline$P$ value & 0.001 & & 0.008 & & 0.17 & & 0.006 & & 0.47 & \\
\hline
\end{tabular}

Table 5. Urinary diversion distribution of study population

\begin{tabular}{lccc}
\hline & Ileal conduit & Neobladder & Total \\
\hline Group A & 44 & 9 & 53 \\
Group B & 44 & 19 & 63 \\
\hline
\end{tabular}

In our retrospective study, postoperative pain score (calculated by visual analogue score) $(P=0.0001)$, analgesic requirement $(P=0.0003)$, length of hospital stay $(P=0.0004)$, duration of surgery $(P=0.0006)$, postoperative paralytic ileus duration $(P=0.0006)$, postoperative wound complication $(P=0.008)$ were less (statistically significant $P<0.05$ ) for group A as compared to group B. But the postoperative hemoglobin drop $(P=0.08)$, the number of units blood transfused $(P=0.189)$, and lymph node yield $(P=0.533)$ were not significantly different statistically (comparable) among the two groups. No statistically significant difference with respect to age and gender in either group was found $(P=0.30, P=0.57$ respectively). For experienced minimal access surgeons, the ablative part of this procedure does not cause major problems but the urinary diversion part of the surgical procedure is challenging and requires more advanced laparoscopic skills ${ }^{[2]}$.

\section{DISCUSSION}

Open radical cystectomy (RC) with urinary diversion (ileal conduit or ileal neobladder) has been the gold standard for treating muscle-invasive bladder malignancy ${ }^{[3]}$. Recently, minimal access approach has been adopted for radical cystectomy in performing laparoscopic RC and robotic RC. As mentioned previously, the minimally invasive approach has various advantages like magnified vision, less blood loss, minimal the postoperative pain, rapid postoperative recovery, less hospital stay and gives better cosmesis ${ }^{[3]}$.

Those who have expertise in advanced laparoscopy have adopted three approaches to reconstruction:

1. Extracorporeal reconstruction through a mini-laparotomy midline or muscle-splitting incision or Pfannenstiel incision. The same incision is used for delivering the bladder specimen and lymph nodes in laparoscopic sacks. We used this approach in our study;

2. A combination of extracorporeal construction of a urinary reservoir and intra-corporeal anastomosis;

3. Complete intra-corporeal reconstruction ${ }^{[2]}$.

A completely intra-corporeal procedure is a technically difficult and time-consuming procedure ${ }^{[4]}$. Manoharan et al. ${ }^{[4]}$ described the Pfannenstiel incision for it.

As for the patients included in group A, out of 53 patients only $4(7.5 \%)$ underwent ileal neobladder, while in group B out of 63 patients 9 underwent ileal neobladder (16.6\%). All the remnant patients in either group underwent ileal conduit urinary diversion [Table 5].

Table 6 demonstrates the final histopathological $\mathrm{T}$ and $\mathrm{N}$ stage distribution of the patients under study.

In contrast to vertical incision, with Pfannenstiel incision, the whole pelvis was easily accessible, without a retractor being applied to the lateral edges $^{[3]}$. Postoperative paralytic ileus duration was significantly higher in patients having lower midline incision, though we adopted the intra-peritoneal approach. The 
Table 6. Final histopathology $\mathrm{T}$ stage and $\mathrm{N}$ stage of study population

\begin{tabular}{lc}
\hline Pathological stage & Number of patients \\
\hline T1 & 16 \\
T2 & 46 \\
T3 & 48 \\
T4 & 6 \\
N0 & 78 \\
N1 & 35 \\
N2 & 3 \\
N3 & 0 \\
\hline
\end{tabular}

technique of robotic-assisted laparoscopic radical cystectomy and neobladder construction or conduit using a Pfannenstiel incision has been favorable. Pfannenstiel incision provides good exposure, facilitating neobladder reconstruction, can be used for specimen retrieval, bowel anastomosis and heals better with a cosmetic scar ${ }^{[4]}$.

Manoharan et al. ${ }^{[4]}$ had conducted a similar study in 2011. He showed that, for those with pfannenstiel incision the mean hemoglobin drop was $1.8 \pm 1$ g per deciliter, while in our study; group A Pfannenstiel incision had $1.75 \mathrm{~g}$ per deciliter mean hemoglobin drop. Mean operating time was $6 \pm 0.8 \mathrm{~h}$ in their study while in our study the mean operative time for group A was $4.3 \pm 1.1 \mathrm{~h}$. According to what was reported by Manoharan et al. ${ }^{[4]}$, in our study there were no intra-operative visceral injuries in group A, but in group B where midline incision was used, there was one case of rectal injury out of 63 patients $(0.01 \%)$, which was managed with a de-functioning colostomy. None of the patients reported by Manoharan et al. ${ }^{[4]}$ had positive surgical margins similar to our study with either group. The mean number of lymph nodes removed was $12 \pm 3$ in their study, by contrast, in our study it was $22.27 \pm 14.32$ for group A having Pfannenstiel incision. The mean hospital stay in their report was 8.5 days, but it was $10.16 \pm 3.39$ days in our study for group A having Pfannenstiel incision.

Raychaudhuri et al.$^{[2]}$ mentioned the work of Puppo, which was a series of laparoscopic RC with transvaginal delivery of specimens. Mini-laparotomy incision was used for the ileal conduit in that series ${ }^{[2]}$. The total operative duration was 6-9 h (in our study it was 4.3-6.9 h) and the hospital stay 7-11 days, but it was $10.16 \pm 3.39$ days in our study for group A having Pfannenstiel incision and for group B it was $13.63 \pm 5.37$ days.

Raychaudhuri et al. ${ }^{[2]}$ also mentioned the work of Denewer who reported a series of 10 laparoscopic salvage (after radiotherapy) RC; this cohort of patients had ureterosigmoidostomy performed through a minilaparotomy ${ }^{[2]}$. Complications (morbidity and mortality) in this study, were comparable with our study.

According to the data from an international registry on laparoscopic RC in 572 patients ${ }^{[3]}$, the mean operating time was $6.2 \mathrm{~h}$ with only $53 \%$ having open neo-bladder. Mean operative time for group A was $4.3 \pm 1.1 \mathrm{~h}$ and that of group B was $6.9 \pm 3.0 \mathrm{~h}$. Most of the midline incision cases were performed in our initial phase of minimal access approach, so we took longer time though the reconstruction time was comparable. The mean length of hospital stay as per that registry was 13 days (range 3-90 days) while in our study group B had similar length of hospital stay $(13.63 \pm 5.37$ days $)$ but it was mere $10.16 \pm 3.39$ days for group A. Intra-operative and postoperative complications occurred in $33(7 \%)$ and $139(28 \%)$ patients, respectively. In our study, postoperative complications for group A occurred in $24 \%$ of patients compared to the results of international registry; for group B complications occurred in $46 \%$ patients, this difference may be because we were in an initial phase learning of minimal access approach ${ }^{[3]}$.

In one recent series of robotic RC, the operating time was reported to be $6.1 \mathrm{~h}^{[3]}$. With a majority of the patients having ileal conduit, the operating time for orthotopic pouch would be much more than the reported mean 
of $6 \mathrm{~h}$. The average operating time in that series was $5 \mathrm{~h}$ for making an open neo-bladder without the use of surgical staplers. The shorter operating time is an important step forward in minimally invasive surgery and it would reduce the anesthesia time and the need for elective ventilation in the postoperative period.

Castillo et al.${ }^{[5]}$ published the complications of laparoscopic RC. Complications (morbidity and mortality) in this study, were comparable with our study, especially that of group A (Pfannenstiel incision group).

Laparoscopic RC and robotic RC provide advantages of minimally invasive surgery with better or at least equivalent outcomes in terms of cancer control. During an initial phase of learning, the operative time will be longer, but over time, there will be an apparent improvement in operational performance, thus reducing the duration of surgery ${ }^{[2]}$.

Grantcharov and Rosenberg ${ }^{[1]}$ observed that transverse incision offers as good an access to most intraabdominal structures as a vertical incision. The transverse incision resulted in significantly less postoperative pain and fewer pulmonary complications. Vertical laparotomy, however, is associated with shorter operating time and better possibilities for extension of the incision. The pooled odds ratio for burst abdomen in the vertical incision group was 2.86 (95\% confidence interval 1.72-4.73, $P=0.0001)$, in our study it was significant too $(P=0.008)$. Grantcharov and Rosenberg ${ }^{[1]}$ concluded that transverse incisions in abdominal surgery were based on accurate anatomical and physiological principles. They should be recommended, as the early postoperative period is associated with fewer complications (pain, burst abdomen, and pulmonary morbidity). Their observations were similar to what we find in our study.

Lunacek et al ${ }^{[6]}$ had very positive experience with the Pfannenstiel incision approach for radical retro pubic prostatectomy. They concluded, this approach provides good exposure of surgical field wound heals well with a cosmetically acceptable scar and facilitates hernia repair through the same approach; if needed, which mimic the results of our study but their study was for an open procedure and the radical prostatectomy, rather than for cystectomy.

Smith et al. ${ }^{[7]}$ conducted multi-institutional prospective randomized trial (RAZOR) comparing open $v s$. robotic radical cystectomy and found that estimated blood loss was significantly lower in the robotic arm, translating into significantly lower blood transfusion rates. Major complications (grade III and above) were similar in both groups. The number of lymph nodes removed was comparable. There was a trend to a shorter length of stay for robotic RC. The results were similar to the group A of our study in terms of complications, estimated blood loss, lymph node yield, and length of hospital stay.

This is a retrospective study, hence the inherent biases of the study design should be considered while interpreting the results. Our data shows we had a bias to perform neobladder with a midline incision in our initial learning curve. This fact might have skewed the data towards significantly high blood loss and duration of surgery in group B (midline incision group). This bias associated with the learning curve (rate of surgeon's progress in gaining experience) should be considered while interpreting the data.

We feel that more advanced disease may make the operation more challenging and potentially longer or with more complications. Higher stage of disease may make the procedure more challenging, but this needs further evaluation and confirmation with future studies. This study was not based on enhanced recovery after surgery (ERAS) protocol.

In conclusion, Pfannenstiel incision enhances postoperative recovery with minimal complications. It has an advantage over midline incision without compromising oncological outcomes. Pfannenstiel can be incision of choice for extra-corporeal diversion. 


\section{DECLARATIONS}

\section{Authors' contributions}

Concept: Ganpule AP

Data collection, compilation: Tak GR

Manuscript writing: Tak GR, Ganpule AP

Manuscript editing: Tak GR, Ganpule AP, Singh AG, Sengar APS, Mohankumar V, Balaji SS, Sabnis RB, Desai MR

\section{Data source and availability}

Data source is available with drgopaltak@gmail.com in excel sheet form.

\section{Financial support and sponsorship}

\section{None.}

\section{Conflicts of interest}

All authors declared that there are no conflicts of interest.

\section{Patient consent}

Not applicable.

\section{Ethics approval}

As it is a retrospective study design, the ethical approval is not applicable in our hospital.

\section{Copyright}

(c) The Author(s) 2018.

\section{REFERENCES}

1. Grantcharov TP, Rosenberg J. Vertical compared with transverse incisions in abdominal surgery. Eur J Surg 2001;167:260-7.

2. Raychaudhuri B, Khan MS, Challacombe B, Rimington P, Dasgupta P. Minimally invasive radical cystectomy. BJU Int 2006;98:1064-7.

3. Shrinivas RP, Dubey D. Technical steps of open radical cystectomy and orthotopic neobladder to achieve the goals of "minimally invasive surgery"? Indian J Urol 2011;27:291.

4. Manoharan M, Katkoori D, Kishore TA, Antebie E. Robotic-assisted radical cystectomy and orthotopic ileal neobladder using a modified Pfannenstiel incision. Urology 2011;77:491-3.

5. Castillo OA, Abreu SC, Mariano MB, Tefilli MV, Hoyos J, Pinto I, Cerqueira JB, Gonzaga LF, Fonseca GN. Complications in laparoscopic radical cystectomy: the South American experience with 59 cases. Int Braz J Urol 2006;32:300-5.

6. Lunacek A, Radmayr C, Horninger W, Plas E. Pfannenstiel incision for radical retropubic prostatectomy as a surgical and cosmetic alternative to the midline or laparoscopic approach: a single center study. Cent European J Urol 2014;67:149-52.

7. Smith ND, Castle EP, Gonzalgo ML, Svatek RS, Weizer AZ, Montgomery JS, Pruthi RS, Woods ME, Tollefson MK, Konety BR, Shabsigh A, Krupski T, Barocas DA, Dash A, Quek ML, Kibel AS, Parekh DJ. The RAZOR (randomized open vs robotic cystectomy) trial: study design and trial update. BJU Int 2015;115:198-205. 